\title{
Visual Enhancement of Service Maps in Logistics Clouds
}

\author{
Michael Glöckner, Björn Schwarzbach, \\ Andreas Barton, André Ludwig \\ University of Leipzig \\ Grimmaische Strasse 12, \\ 04109, Leipzig, Germany \\ Email: \{gloeckner, schwarzbach, barton, \\ ludwig\}@wifa.uni-leipzig.de
}

\author{
Bogdan Franczyk \\ University of Leipzig, \\ Grimmaische Straße 12, 04109, Leipzig, Germany \\ Email: franczyk@wifa.uni-leipzig.de \\ and \\ Wrocław University of Economics \\ Komandorska 118/120, 53-345 Wrocław, Poland
}

\begin{abstract}
Logistics and its involved parties are nowadays faced with demanding challenges, in order to fulfill their customers' needs. Hence logistics service providers are constrained to cooperate with each other, which leads to the challenge of 'understanding' each other's service descriptions and integrating the strongly differing IT-systems. An emerging approach to solve this problem is the operation of cloud platforms. Main tasks are service retrieval and composition. However, a suitable visualization is needed to generate a high user acceptance. With the service map concept a first step is taken, that certainly needs further improvement. This paper briefly gives an introduction to general information visualization and analyzes the suitability of several approaches for improving the service map concept with regards to different scales of measurement. After their comparison a general guideline for fostering the visualization concept is derived. Objective is the increase of information content while keeping an intuitive usability.
\end{abstract}

\section{INTRODUCTION}

$\mathbf{T}$ RADITIONALLY, the field of logistics is mainly dominated by logistics service providers, which are characterized as small and medium-sized enterprises (SME) [1]. Fig. 1 shows the distribution of logistics companies in the European Union (EU) categorized by the number of employees, for the year 2011 .

In the last few years, but especially since the establishment of the European Union (EU), these SME are constantly facing new challenges. The abolition of traditional trade barriers and the simplification or even elimination of customs regulations led to a specialization of certain geographical regions in the field of production. Another problem for these companies is the strong wage gap between Asian countries and the EU and North American countries, which results in the manufacturing industry migrating resources to these countries. This wage gap is also existent within the EU itself, caused by constant expansion and integration of new EU member states. For instance, in 2004 ten new member States (Cyprus, the Czech Republic, Estonia, Hungary, Latvia, Lithuania, Malta, Poland, Slovakia and Slovenia) joined the EU. At that time these new

The work presented in this paper was co-funded by the German Federal Ministry of Education and Research under the project LSEM (BMBF 03IPT504X) and the CENTRAL EUROPE programme co-financed by the ERDF under the projects LOGICAL and ESSENCE.

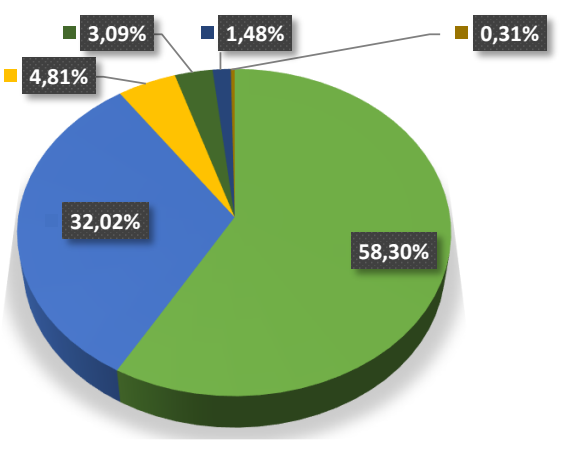

number of employees 0 to 1

- 2 to 9

10 to 19

- 20 to 49

- 50 to 249

- 250 and more

Fig. 1. Distribution of logistics companies in Europe categorized by number of employees[1]

member states had significantly lower average wages than the other incumbent member states. As a result, a non-minor number of manufacturing companies moved their locations to the south-eastern borders of the EU. This - in combination with other factors - leads to the fact that the supply chain of individual companies and company networks is stretched and thus less predictable. One of the major resulting challenges for LSP is to meet the needs of their customers furthermore [2], [3].

To cope with this challenge the division of labor and outsourcing are suitable concepts, as they provide flexibility as well as the possibility for SME to participate in larger networks. Hence, LSP have to cooperate with each other to fulfill their customers needs [4]. The most basic level of reliable networking is to publish the offered services, in order to combine them to more complex logistics services. However, by publishing the services further challenges arise. As LSP usually got a varying quality and style of service description (i.e. based on different description languages, emphasizing different aspects) as well as a wide range of implemented ITsystems (e.g. from SAP down to handmade MS Excel-sheets), an integration of information flow is hard to realize in an ITsupported way [5], [6]. In order to overcome this challenge 
cooperating partners should make use of a common platform or cloud platform, respectively.

Project ESSENCE, an project within the Central Europe program - co-financed through the European Regional Development Fund (ERDF), aims to create an information and communication technology (ICT) platform. The general objectives of this platform are an increased effectiveness of transports, reducing its environmental burden, and the neutralizing of current deficiencies in SME to increase their productivity and competitiveness. ESSENCE strives to establish an ICT network that SME are encouraged to use - free of charge to manage their logistics and optimize their supply chain by designing their own business networks. [7] Finding the right partners for fulfillment of atomic process steps is a difficult task. In the past, several scientific approaches to this problem have been developed.

To be a connecting tool between the participating LSP, the main functions of such a platform comprise retrieval and composition functions for services. Those functions are to be customized in an easy-to-understand and intuitive way in order to gain a high user acceptance. Visualization is outlined in literature as important aspect to aid users in exploring, understanding, and analyzing data through progressive, iterative visual exploration, especially in data analysis applications of business informatics related fields (e.g. visual analysis of business data, scientific data, images and videos, auction data, search results) [8]. Hence, the importance is given of visualizing service retrieval, composition and management in heterogeneous logistics clouds in a sophisticated way. One strategy is a combined approach of catalog and modular service construction system - the logistics service map (SM) [9]. Since the state of the art of the SM concept is still in draft mode, experiences with SMs still contain potential for improvement. The current structure is quite simple and the offered range of functions and displaying possibilities is way too small.

After the introductory part the basic principles of information visualization are being presented. Section three gives an overview on the current state of cloud platforms and provides basic definitions of terms such as service, process activity and process model within the context of project ESSENCE. The reader gets an impression on how the ICT platform and underlying principles are working together. In addition to that, the concept of SMs will be introduced to the reader together with an adaption to the logistics sector (logistics SM). In section four several approaches on increasing the information content of the SM by applying different combinations of scales of measurement and visual strategies are proposed and analyzed. Finally a comparison of the approaches leads to a guideline for an enhanced visualization for SMs. The paper is concluded by a short summary and future research prospects.

\section{INFORMATION VISUALIZATION}

As already outlined information visualization is an important aspect in business information systems. A comprehensive design study methodology is proposed in [10] with different phases from preliminary general preconditions (e.g. general design science knowledge acquirement, casting of all stakeholders) over core activities (e.g. problem analysis, developing alternatives, implementation and deployment) to outwardfaced validations. As they assume the design process for information visualization done by specialized domain-foreign designers and the authors only focus on core activities and claim to have sufficient knowledge in the logistics and cloud domains from previous research and development projects with partners from industry and service sector, the most important and influencing parts for our purpose are the following. When developing information visualization the essential points are at first a sufficient range of possible solutions to be considered (i.e. different visual encoding and data abstraction). Afterwards those solutions are to be filtered down to a narrow proposed selection. Further [10] advise to refer on generally accepted design principles and guidelines. The major points are briefly introduced in the following paragraphs.

Commonly accepted and generally applied aspects on design principles for information visualization are outlined by [11]. First, he summarizes 'perception and purpose' as important aspects with appropriate visual affordance (e.g. differentiating contrast of brightness easier for human eye than contrast of colors or getting a common basis for optical comparison), identifying the right objectives, goals and tasks (e.g. setting right basis for comparison) and aesthetics (e.g. complementary colors or sectio aurea (golden ratio)). As these aspects often collide Dix further highlights 'interaction' as a solution. For this aspect drill down and hyperlinks, overview and context are highlighted as well as dynamization (i.e. changing parameters and representations, temporal fusion of different information views). Moreover, the 'visualization mantra' of [12] is mentioned with: "Overview first, zoom and filter, then details on demand". 'Information scent' [13] is a term describing the intuitiveness of handling information. In order not to click through every possibility in a matter of trial and error even if total information is not available, the user always needs a kind of clue to 'scent' on where to seek next for suitable, more detailed information. With focusing on the wider context, the perspective on different stakeholders and the resulting effects of information visualization shall be taken into account (e.g. analysts, customers, senior management). Other literature analyzes the capacity limits of attention and the subsequent influence on information visualization [14]. They conclude grouping similar objects in some cases as a powerful guideline. Moreover a proper legend or key, as well as a reduction of categories actually displayed help working with visualized information. With regard to capacity limits of attention the number of nominal categories should be dedicated to data with high dynamic range or different categories. Generally they tend to a smaller number of categories and colors to keep the user focused.

In [8] an extensive literature review on challenges of information visualization is conducted. As a conclusion of their paper they summarize the following five major technical challenges: 
1) Usability: As the user of a system is inevitably involved in the visualization and toolkit system to accomplish its analysis, retrieval and composition tasks, the visualization shall support the user in an intuitive and efficient way.

2) Visual Scalability: Following the former mentioned visualization mantra [12], the capability of effectively displaying large data sets is an important challenge. The core part is constituted in data reduction techniques [15], as the amount of data easily exceeds the display capacities.

3) Integrated Analysis of Heterogeneous Data: The aspect of handling data from multiple sources is marked as one of the biggest challenges.

4) In-Situ Visualization: This point mainly focuses on the effective visualization of streamed data (e.g. in social networks like twitter) and how to share same processor and memory space in order to synchronize data processing and visualization task. As the concepts presented in this paper do not deal in any aspect with streamed data, this point does not hold any relevance for our purpose.

5) Errors and Uncertainty: Errors in data sets (e.g. noisy and inconsistent social media data, imprecise data from sensors) and/or resulting errors by data transformation are to be displayed to the end-user as well, in order to strengthen the truthfulness of visualization. As homogeneous data from a proven source is assumed, this point does not apply big relevance to our purpose.

The visualization pipeline is a technical infrastructure fostering information visualization is introduced by [16] with reference to [17]. However, they outline the concept, which is applied for visualizing service-oriented architectures (SOA), the concept could be abstracted and thus, also be transferred to other similar context. The four sections of the pipeline contain a typical ETL-process (i.e. the first three sections) extended by a reporting in terms of a final graphical representation. At first, heterogeneous data from different sources (e.g. UML, BPMN, $\mathrm{XML}$ ) is extracted. Secondly, a transformation to a consistent and semantically correct form is conducted to gain the relevant meta data. Thirdly these data are loaded in a central repository as well as the pre-processed data from the repository is loaded to the next section, tailored by stakeholders' configuration (e.g. pre-defined views for different stakeholders are possible). Fourthly and finally, the graphical representation is processed from the pre-processed data from the repository taking the stakeholders' configuration into account.

Summarizing, the phases of developing alternatives and their evaluation are important and to be solved using general design guidelines. The challenges of usability and scalability are the emphasized designated requirements for the visualization approaches of the SM. As the paper focuses on visualization more in cognitive than in a technical way, only the last two sections of the visualization pipeline are considered.

\section{Logistics Clouds and Service Maps - State of THE ART}

After giving a briefly discussing information visualization and its importance in business information systems the paper now proceeds by introducing a current approach of a cloud platform that could be used in the logistics sector. After introducing the SM concept as well, general visual disadvantages of both concepts are outlined, that are to be solved during the latter progress of the paper.

\section{A. Logistics Cloud}

At this point, the focus is on describing an existing approach - as a representative for cloud platforms in general - as detailed as possible within the restricted frame of this paper.

1) Working Definitions of ESSENCE: The ESSENCE platform [7] encourages SME in exploring the use of customized eServices that give logistics support their business. Supply chains are modeled by application of three different entities. Those entities are named: process activity, process model and service. In order to fully understand the operation principle of ESSENCE the aforementioned terms have to be defined in a short way.

Process activities are the smallest units in the ESSENCE supply chain. They are single atomic actions, described by the values 'Name', 'Description' and 'Type'. While the meaning of 'Name' and 'Description' are self-descriptive, 'Type' subdivides the activities into four distinct categories. 'Standard activities' are to be fulfilled at the side of the activity creator. It's counterpart - the 'At customer'-activities are done at the customers side. 'Latency activities' are fulfilled either at creators or customers side. Each of those latency activities has a predecessor whose completion is obligatory. The fourth category is being shaped by the so called 'custom process activities'.

Further, a process model results from the combination of one or more of those process activities. Similar to the aforementioned process activities these models are specified by a 'Name' and a 'Description' which are free-text fields. The values 'Duration', 'Quantity' and 'Cost (EUR)' are assigned additionally to each process activity enclosed in a process model. Furthermore, process activities within a model can be sequenced by using the field 'Sorting'.

The superior entity service consists of one or more process models and is specified by free-text fields containing 'Name' and a 'Description' (each twice, both in local and in common platform language 'lingua franca'). In addition to that a service could also contain 'Functional parameters', a 'Classification', 'Attachments' and 'Dependencies'.

2) ESSENCE Operation Principle: After having introduced the terms service, process model and activity in the context of the ESSENCE platform this section shows its current operation principle [7]. The workflow for designing process models is shown in a roughly simplified mainstream manner without considering any exceptions or irregularities, which is illustrated in Fig. 3.

After the initial registration process, which is approved by the platform provider, the user is able to log in. In order to publish its company's service offer on the platform the service list view is used. Each service needs a name and a short description in local language and lingua franca as well. 


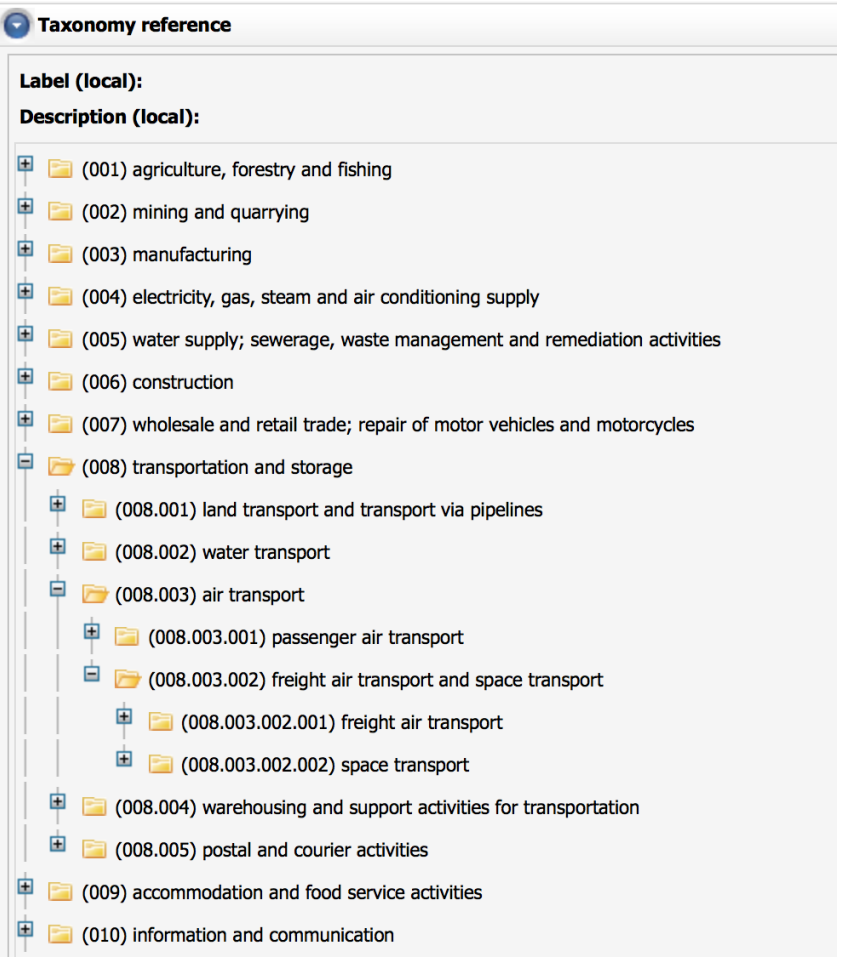

Fig. 2. Example of the taxonomy used by the ESSENCE platform.

In addition a service can have one or more parameters, e.g. number of packages of the shipment. Those parameters are used during process modeling to transfer information from one activity to another. Services can also be categorized by the ESSENCE taxonomy. This taxonomy is a tree with industrial sectors as nodes and leaves. Fig. 2 shows an example of the taxonomy tree. In addition services can have attachments and depend on other services.

To create process models the user first needs to create atomic activities in the activities list view. Therefore the user has to set a name and a description for the particular activity. After creation of this activity the user has to assign one or more supplier services to this activity. Such supplier services are alternatives, i.e. during process model execution one of the alternatives is selected.

After defining the process activities a process model is created as a orchestration of the process activities. An example of a process model consisting of three activities is shown in Fig. 4 with local language Hungarian.

During the whole process model creation the most time consuming and important step is the selection of the service suppliers and their services for the activities. To add a service of a service supplier to an activity the user first has to select the supplier and then the service of the supplier. There is no possibility to find similar services of different suppliers or filtering of services for some criteria yet. Nonetheless such a functionality would be very helpful and result in a higher usability and subsequently user acceptance. A first attempt towards such a functionality was done by implementing the taxonomy of services mentioned before. This taxonomy enables the selection of services according to their purpose. The main disadvantage of this approach is that although the taxonomy is very fine-grained it is not flexible as it offers just one dimension to categorize the offered services.

As the number of services within a network is growing the challenge of finding and selecting appropriate services is growing more than linear. Hence, an advanced approach for visualizing, filtering, revealing and combining the services of a cloud platform is needed.

\section{B. Service Map Concept}

As already outlined in the context of collaborating logistics service providers, participants are faced with the major challenge of a lack in standards for service descriptions (activity descriptions, respectively) and IT-systems in logistics [5]. Hence, in a network of logistics service providers a wide range of description types with differing wording and formats arises. Representative survey conducted within a network of small and medium logistics enterprises motivating this topic can be found in [6]. To solve this problem cloud platforms - like the introduced ESSENCE platform of the former subsection - have been developed, to support the main functions of presenting the available services (or activities, resp.) of a network (catalog function) and combining them to complex composite services (modular service construction system function), in a way that is commonly accepted by all network participants. As current approaches lack in providing a suitable visualization (e.g. the ESSENCE platform, see also Fig. 2) sophisticated approaches are needed. This issue is solved by the concept of the service map (SM). As the concept is based on another set of wording definitions the according terms of the ESSENCE platform are always given in parentheses.

In literature exists a wide variety concerning the SM concept. Either (1) the term 'service map' is used and also the functionality meets (partly) the requirements mentioned above or (2) the term is used, but a different substantial functionality is addressed or (3) the term is not used, but the described concept (partly) includes functionality for the mentioned purpose.

The approaches of (1) [18], [19], [20], [21] are situated in a wide range of scientific fields. The highest relation to the described context could be found in [18]. The SM provides an overview of existing service portfolio of a company (in the field of financial industry). Main objective is the merging and outsourcing of different companies' portfolios or business models and the related IT-systems, respectively. The catalogrelated objective of service retrieval and the construction system-related objective of creating atomic and composite services are not addressed. In [19] the authors categorize mobile services and apps with the help of a scale involving dimensions of 'customer needs'. The revelation of 'empty spaces' within the maps helps identifying yet not satisfied combinations of different customer needs that could be covered by innovative new services or apps. The authors of [20] propose an XML- 


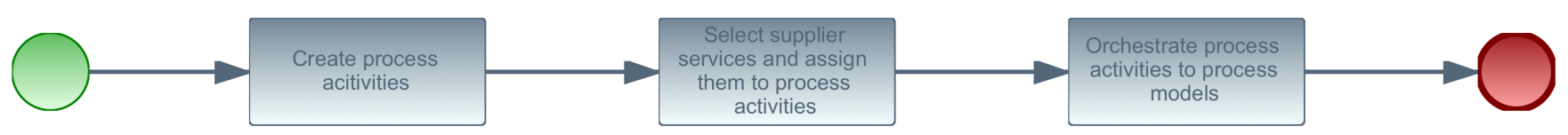

Fig. 3. BPMN diagram of the ESSENCE workflow for designing process models.

\begin{tabular}{|c|c|c|c|c|c|c|c|c|c|c|}
\hline \multicolumn{11}{|c|}{ General information } \\
\hline \multirow{2}{*}{\multicolumn{3}{|c|}{$\begin{array}{l}\text { Process model name: } \\
\text { Process model description: }\end{array}$}} & \multicolumn{8}{|c|}{ Csipke folyamatmodell } \\
\hline & & & \multicolumn{8}{|c|}{ Csipke keszites folyamatmodell } \\
\hline \multicolumn{11}{|l|}{ Save } \\
\hline \multicolumn{11}{|c|}{ Process activities } \\
\hline Select & \multicolumn{3}{|c|}{ Name } & \multicolumn{2}{|l|}{ Description } & Type & Quantity & Duration & \multicolumn{2}{|c|}{ Sorting } \\
\hline$\square$ & \multicolumn{3}{|c|}{ csipkeveres } & \multicolumn{2}{|l|}{ csipke keszites, festes } & Standard & 1 & 12 & & 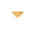 \\
\hline$\square$ & \multicolumn{3}{|c|}{ Csipke csomagolas } & \multicolumn{2}{|l|}{ Csipke csomagolasa } & Standard & 1 & 2 & 2 & $\Delta \nabla$ \\
\hline$\square$ & \multicolumn{3}{|c|}{ Packaging } & \multicolumn{2}{|l|}{ Goods packaging and delivery } & Standard & 1 & 0 & 3 & $\Delta$ \\
\hline & & & & & $\mathrm{N} \| \Delta \rightarrow \mathrm{B}$ & & & & & \\
\hline Add ac & tivity & Preview & Delete & & & & & & & \\
\hline
\end{tabular}

Fig. 4. Example of an ESSENCE process model with 3 activities.

\section{Logistics Service Map}

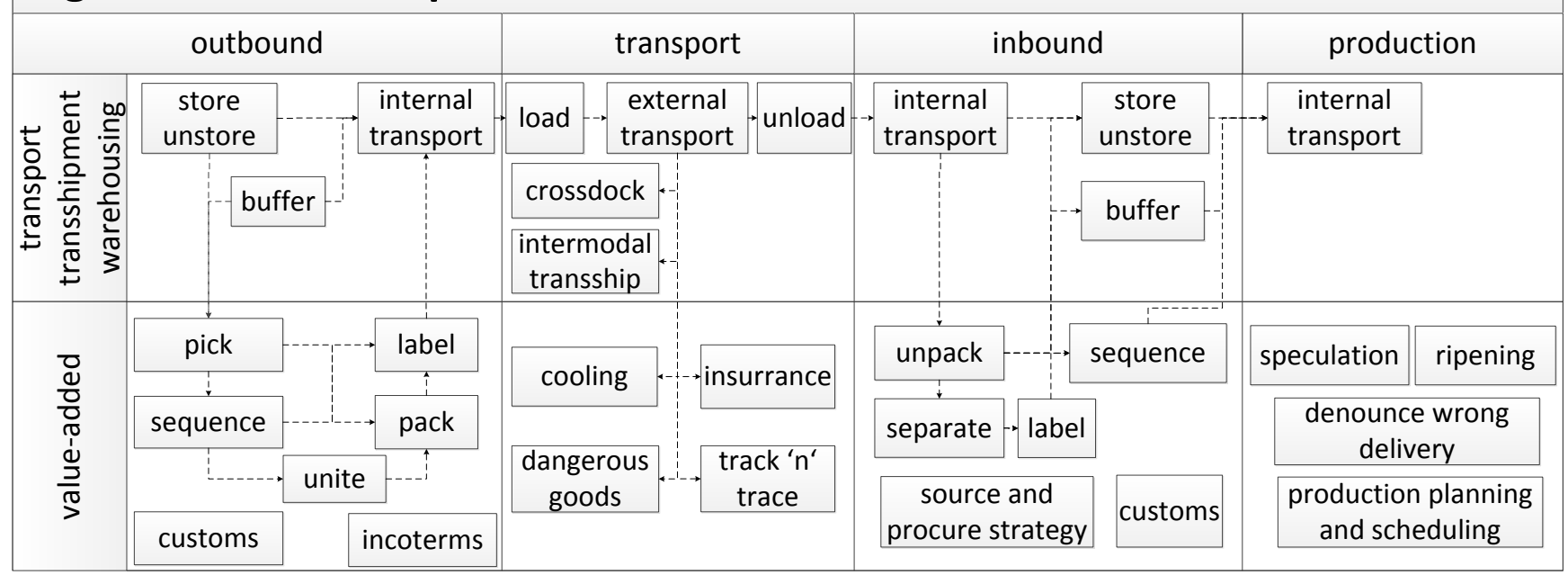

Fig. 5. Exemplary service map catalog with two dimensions: 'classic logistics function vs. value-added' and 'stage-specific'. Dashed arrows mark suggested services for composition.

based approach for structuring and categorizing services with new association and combination operators based on XMLtags. However, the approach lacks in maturity because a clear usage concept and a proper visualization is missing. [21] present a mobile data management approach. With obtaining a detailed view of available networks and their inherent capabilities, attributes and offered services in the surrounding of a mobile device. However, their categorization pattern is spatial-based and the combination functionality is missing.

The case (2) [22] addresses a SM for mapping or matching, respectively, of Quality of Service (QoS)-classes. The approach deals with data quality in heterogeneous networks consisting of several network technologies. The goal is a mapping of performance parameters of the different technologies. Neither overview of services nor building composite services is focus of the approach.

The first concept of (3), the 'service portfolio management framework' [23] combines service science with portfolio management. Hence its focus lies on strategic service management and the construction system functionality is missing. In [24] the 'business capability map' is introduced. Like [18] it focuses on comparing different companies concerning their 
capabilities of serving special tasks in order of outsourcing but does not provide retrieval or construction function.

Summarizing, an approach combining both, the catalog and the construction function, is not a part of the above mentioned concepts. Subsequently, the concept of the SM introduced by [9] addresses the challenge by combining the two functions. First a catalog of all available services and process activities is provided, that could be categorized by the user's needs in different abstraction layers. As shown in Fig. 5 a graphical representation with two spatial dimensions for the user-chosen categories simplifies the interaction for the users when searching for services or process activities. Hence, service retrieval is enhanced and done in an intuitive way. Further, the concept includes a modular service construction system in order to combine atomic activities to composite services. With this approach, the network participants are supported in retrieving services in different use cases. Firstly, adding a new service provider (supplier) to the network and matching its offered services to the existing set of services by just adding the new service provider to the provider list of the particular services. Secondly, developing a new composite service (process model) to meet a specific customer's need by selecting and composing services from the SM. Service-specific information and attributes can be displayed when changing the selected granularity to a more detailed level to foster planning and monitoring. Moreover, the unique standard of the used set of services within a network and the visualization foster a precise mediation and communication between all stakeholders during the whole service life-cycle. Thirdly, finding compensational service (activity) or provider (supplier), when realizing the urgency for replanning or elimination of errors because of unpredictable disturbance in the network. Consequently, a $\mathrm{SM}$ is considered to be a core element of a service-oriented engineering and management platform or cloud-based service platform, respectively.

As the SM concept is an still evolving draft, certainly further development is needed to exploit its full potential. Especially, service retrieval is in the focus as this function is limited by two spatial dimensions and realized by 'highlighting categories' (1. displaying them at all, 2. reduction of displayed services by adding another category). The following section gives a more detailed introduction of the shortcomings and potential approaches for improvement.

\section{Visual Enhancement of Service Maps}

The idea of a SM shown in the previous section has two points with potential for improvement. This section introduces these two ideas in detail and concludes with a brief guideline for the visual enhancement of SM concepts.

\section{A. Preliminary Consideration}

The first enhancement could be provided with the possibility of having more than only two dimensions displayed in the $\mathrm{SM}$ at a time. This point gains more importance with an increasing number of services available in a network that are to be displayed. Since existing approaches of SMs are only two-dimensional, the benefit of using a SM is limited in terms of category quantity. Especially with a high amount of services available in a network, the service representation lacks in clarity due to this limitation. The possibilities of integrating further categories by adding more dimensions are needed to improve the work with the SM.

Another shortcoming is that the SM categorization is fixed and cannot be changed on demand in runtime. An enhancement could be reached with the possibility of adapting the categories of the SM on the fly according to the current user needs. This would provide the user with an even more intuitive experience on the service retrieval and composition.

As summarized in the section concerning information visualization, the paper follows the methodology of [10] and focuses on developing alternatives, which are evaluated with reference to the main requirements 'usability' and 'scalability' [8]. As categories are taken into account for service retrieval and each category follows a particular type of scale, the following four scales of measurement [25] are considered. A nominal scale is able to display distinct named values with no natural order at all. An ordinal scale capable of displaying nominal values that can be brought to a natural order but have no measurable distance metrics between two values. A interval scale is capable of displaying quantitative numbers, which can be naturally ordered and own a distance metric between the values and a ratio scale as a special characteristic of a interval scale that includes a origin of ordinates.

\section{B. Adding Further Dimension}

Via brainstorming the authors and partly the ESSENCE consortium figured out the following attempts, that are compared and discussed in detail: (1) spatial dimension, (2) colored dimension and (3) shape dimension.

1) Spatial Dimension: As the current version of the SM only displays two dimensions, adding another spatial dimension is an obvious option. [16] figured out a lack in understanding and representing SOA (service-oriented architectures) (i.e. 3 layer: business-process, service interface, application) as well as the inherent intra-layer and cross-layer connections and dependencies. Reason to this is an unappropriate visualization. Outlined disadvantages of two-dimensional visualization are either overlapping connection lines or a lack in displaying different diagram types simultaneously. Hence, they summarize a loss of context with a growing number of information units and thus, propose a three-dimensional approach to visualize processes and their related services modules and classes. Drawing an analogy between the visualization of a SOA and a $\mathrm{SM}$, a common evidence can be found for the requirement of an appropriate visualization with a growing number of services and inter-relations to be displayed and hence, adapt this idea to the SM.

Adding a spatial dimension to the SM leads to two possible approaches: either bringing the three-dimensional SM on a two-dimensional output device like in computer games (i.e. ordinary screen) or an three-dimensional output device (i.e. virtual reality environment, 3D display). As the latter one puts 
high requirements on technical infrastructure it proves rather unsuitable for the usage in SME. As a consequence, the usage of a two-dimensional output device is proposed. Advantages are the intuitive usage and the possibility of appealing views. Whereas the main disadvantages are a difficult navigation within the SM, a high development effort and the limitation of only one further dimension.

2) Colored Dimension: Another approach of adding further dimension is visualizing categories by colors which means that the services that are shown in the SM will be colorized with an corresponding color.

Tufte states, "The fundamental uses of color in information design [are]: to label, to measure, to represent or imitate reality, to enliven or decorate." [26]. Further, he provides some basic guidelines on how to use colors for visualization of information. Small color spots on light gray background highlight the information. Such spots should be of no more than 20 to 30 colors found in nature, else negative returns would be produced by the colors. It is also important to use colors of the same hue and with maximum differentiation (e.g. yellow, red, blue, black).

"Color itself is subtle and exacting. And, furthermore, the process of translating perceived color marks on paper into quantitative data residing in the viewer's mind is beset by uncertainties and complexities. These translations are nonlinear (thus gamma curves), often noisy and idiosyncratic, with plenty of differences in perception found among viewers (including several percent who are color-deficient)." [26] As already stated before [14], the usage of color as a further dimension shall be taken very carefully.

The first step for using color coding is to identify the scale of measurement of a dimension. Because of the different characteristics of the different scale of measurement it is necessary to use different colorization models for different scales of measurement. A commonly used approach for color coded scales is the color gradient as shown in Fig. 6. A color gradient either consists of two colors for a minimum and a maximum value or includes intermediary colors for intermediary values. To determine the exact color of a specific characteristic service within a category it is necessary to compute the characteristic's distance to the end points of the scale. The color of the service is proportional to the distance to the ends of the color gradient. Since it is necessary to compute a distance such a color gradient can only be applied to interval and ratio scales. A widely used color gradient coding is the red to green gradient where red stands for poor values and green stands for appreciated values, e.g. referring to a logistics delivery schedule whereas red stands for all deliveries out of schedule and green stands for all deliveries in time, for intermediary ratio of $50 \%$ of deliveries in time a yellow colorization would be used as it is situated in the center of the gradient. Red-green-gradients are only suitable for assessment category dimensions referring to the maxima poor and optimal, because people always perceive red as bad and green as good due to the extensive common usage of this coding. Hence, for other categories it is obligatory to select other colors as endpoints of the scale. Categories presenting the intensity of one distinct characteristic in interval or ratio scales are likely to use a gradient from 'non-colorful' color like white to a colorful one. Again, the usage of green and red is to be considered carefully.

Another important step is the selection of the color space for the gradient. RGB and HUV are well used and well known color spaces. The main problem with these color spaces is that the perceived distance between two colors is not the same as the computed distance, hence RGB and HUV are not a good choice for the gradient. A color space addressing this problem is the CIE-LUV color space where the distance of all colors is proportional to the perceived distance [27] For dimensions of ordinal scale it is possible to use a color gradient with uniform partitioning. This results in a clear visualization of the order of the categories to the user. For nominal scales there is no method to assign special colors to categories of the dimension. If the CIE-LUV color space is applied the colors of all the categories should have a maximum distance to each other to ensure an optimal discriminability. To provide the user of the $\mathrm{SM}$ with a basis for interpretation of the categorization colorcoding it is necessary to show a legend giving information on the meaning of the colors and the scale.

However, the main problem with color coding of categories and their particular characteristics is a lack of a common way of perception. Every person perceives colors in a different way. There are people with color vision deficiency, e.g. monochromacy, red-green or blue-yellow. Those people will possibly not be able to perceive the information that is color coded. Hence, an ideal solution provides an option to disable color coding or to use another coding instead.

3) Shape Dimensions: Another approach is to add a dimension by dedicating distinct shapes to different categories. In the beginning, the proposed forms related to geometrical shapes (e.g. circle, ellipse, rectangle, polygon, star-shaped, heartshaped, cloud-shaped and arrow-shaped). For instance, one approach included a matching of shape and semantic of a service (e.g. transport services were presented with an arrow, while warehouse services were illustrated by pentagons in house shape). Unfortunately, preliminary tests revealed an enormous lack in usability. In other cases it was much more difficult to illustrate dimensions with appropriate shapes e.g. presenting a characteristic with a $n$-cardinality with the assistance of an $n$-sided polygon or visualizing dimensions with an ordinal scale. Further disadvantages are an unfavorable behavior in line break of labels as well as a tricky cognitive differentiation when a category with a multitude of characteristics had to be represented by a multitude of different shapes (high range of different $n$-shaped polygons). However, as the matching of shape and service semantics appears to be an appealing and useful approach, the decision was made to transform this idea to a more practical level. The classical rectangle-shape for services was retained and the shape dimension is integrated by reducing forms to pictograms that are to be displayed within or next to the rectangles as shown in Fig. 7. Another finding integrated in the concept is that this dimension is only useful 


\section{Logistics Service Map}

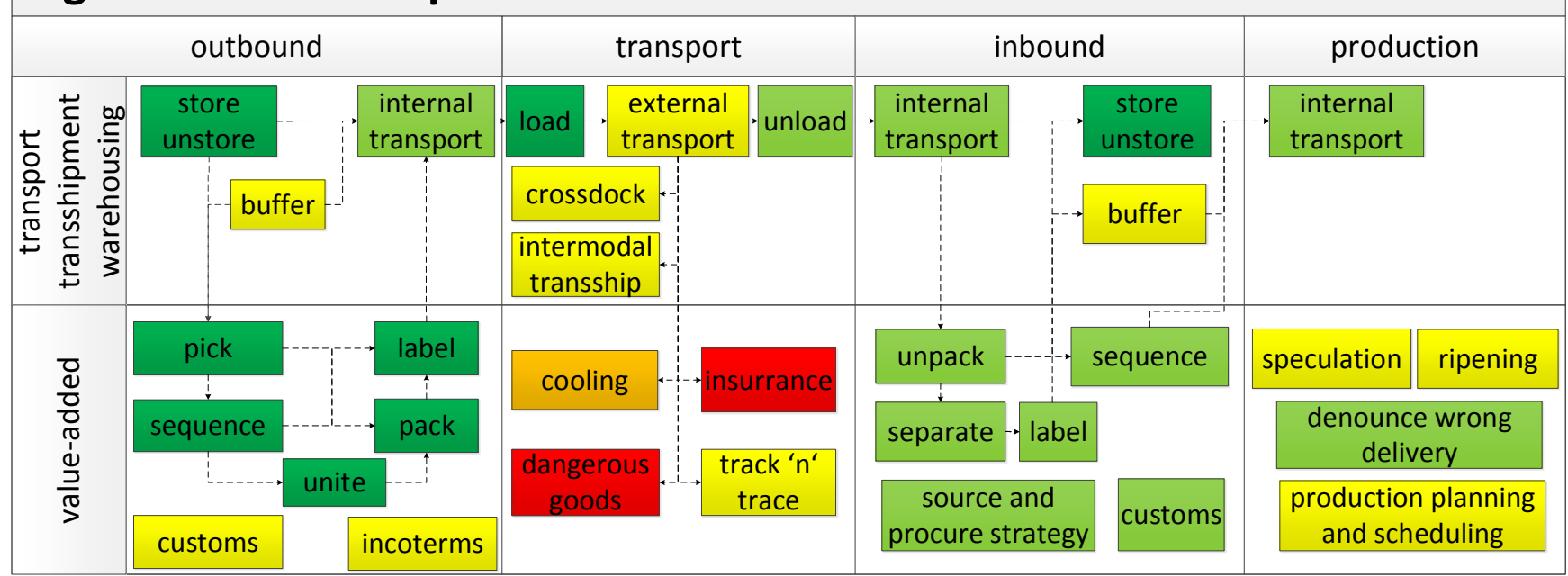

poor

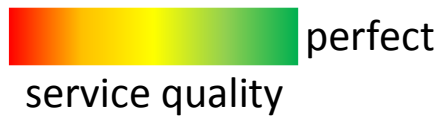

Fig. 6. Example of color gradient dimension for service map catalog.

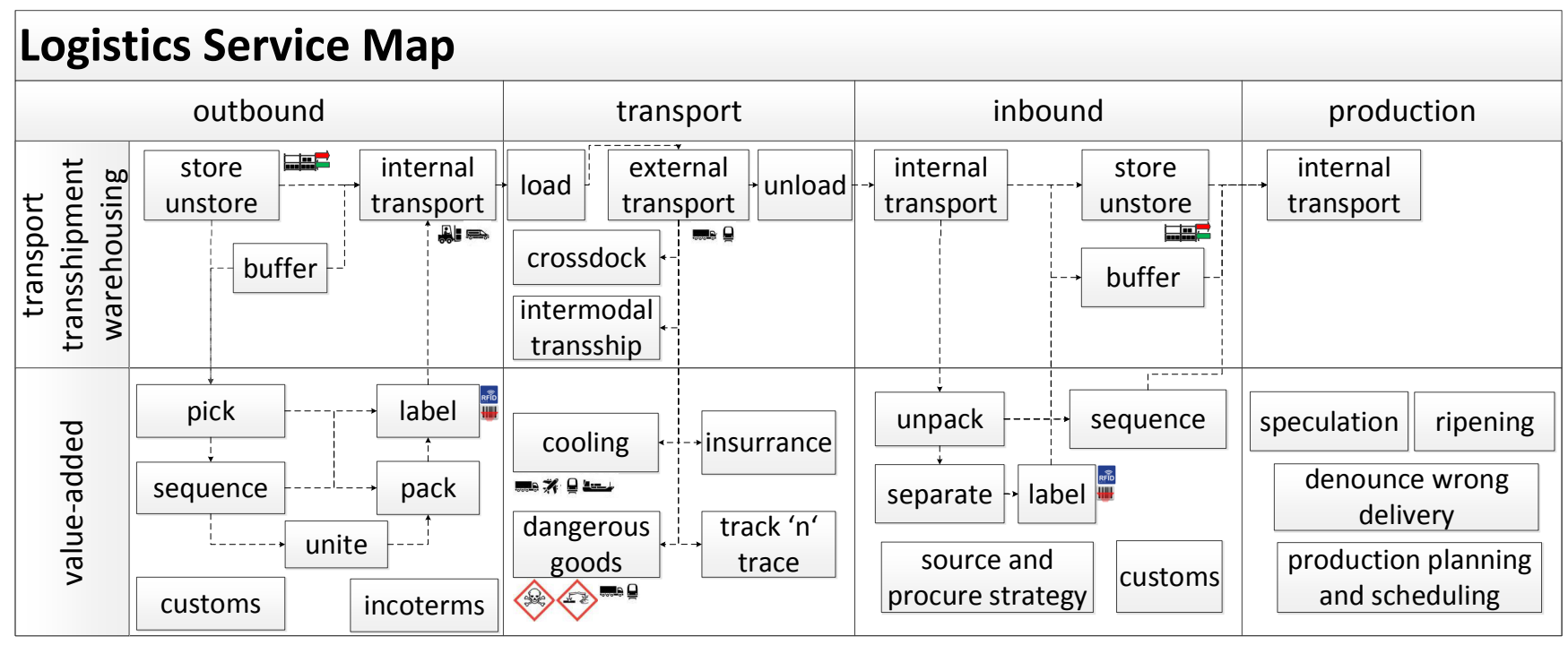

Fig. 7. Example of pictogram dimension for service map catalog.

for categories, which rely on a nominal scale.

Results of short interviews-conducted in our research group and partly the ESSENCE consortium-for comparison are shown in Table I. Spatial dimensions, i.e. 3D-SMs, were rated very poor. The only possible usage is for nominal and ordinal scales. The navigation within the 3D-space of the SM was the main problem for most of the participants in the interview. Spatial dimension do not scale well with higher number of services since they just add space in the third dimension but this space is limited for every characteristic because of clarity. Most of the participants of the interviews stated that color dimensions are suitable for ordinal, interval and ratio scales because they are easy to understand and can be processed pre-attentively which leads to intuitive usage. Color dimensions are also suitable for displaying nominal scaled categories but only with limited number of characteristics, as a too colorful impression only leads to user confusion. All participants agreed that pictograms are very suitable for nominal scales. As pictograms are not very capable in displaying order or metric, other scales than nomial are not suitable. Pictograms are appropriate for small as well as for high numbers of services. A possible problem of pictogram 
TABLE I

COMPARISON OF USABILITY AND SCALABILITY OF PROPOSED APPROACHES WITH VALUES FROM 0 (POOR) TO 10 (OPTIMAL) (N—NOMINAL SCALE, O-ORDINAL SCALE, I-INTERVAL SCALE, R-RATIO SCALE)

\begin{tabular}{|l|c|c|c|c|c|c|c|c|l|l|}
\hline \multirow{2}{*}{ Approach } & \multicolumn{5}{|c|}{ Usability } & \multicolumn{3}{|c|}{ Scalability } & \multirow{2}{*}{ Advantages } \\
\cline { 2 - 9 } & N & O & I & R & N & O & I & R & Disadvantages \\
\hline Spatial & 6 & 7 & 2 & 2 & - & - & - & - & $\begin{array}{l}\text { intuitive usage, adds space for cate- } \\
\text { gories instead of using the exitsing } \\
\text { space }\end{array}$ & $\begin{array}{l}\text { high development effort, only one } \\
\text { further dimension, difficult naviga- } \\
\text { tion }\end{array}$ \\
\hline Color & 5 & 8 & 9 & 8 & 6 & 7 & 8 & 8 & $\begin{array}{l}\text { easy to understand, pre-attentive } \\
\text { processing }\end{array}$ & $\begin{array}{l}\text { problems with color blind people, } \\
\text { unclear if used with nominal scale } \\
\text { with many categories }\end{array}$ \\
\hline Pictogram & 10 & 2 & 0 & 0 & 9 & 2 & 0 & 0 & $\begin{array}{l}\text { ideal for nominal scales, easy to } \\
\text { understand, pre-attentive process- } \\
\text { ing }\end{array}$ & $\begin{array}{l}\text { not suitable for other scales than } \\
\text { noffort }\end{array}$ \\
\hline
\end{tabular}

dimensions is that there is a need for particular pictograms for every characteristic of the scale.

The paper concludes the following guideline: the usage of pictograms for nominal scales and color dimensions for ordinal, interval and ratio scales is proposed. If there is the need of having multiple ordinal or nominal scales then a spatial dimension could be taken into consideration. However, with two spatial dimensions and further added color dimension and pictograms there are four dimensions in total displayed in the SM. Regarding the capacity limit of attention, four dimensions are sufficient as too much categories (and the related dimensions, respectively) are counter-productive and reduce clarity and usability.

Referring to the visualization pipeline concept [16], the integration of this guideline is proposed for the third section where a stakeholder could choose options for the resulting visualization due to its requirements.

\section{Dynamization}

Despite the possibilities of codings for further dimensions of services, the other problem remains: if there are too many services in one category to be displayed all at once. To address this problem the visual information seeking mantra "Overview first, zoom and filter, then details-on-demand" [12] can be applied. Hence, an aggregation of the individual services (and their characteristics and visualization, resp.) is needed. The result is an overview of this category, which is a compromise between information content and possible perception. Best practice depends on the type of the chosen dimension coding. If color coding applies, one approach for $n$ distinct characteristics in a category is to have $n$ equally sized parts of the category with a distinct color. Another approach is to size the colored parts proportional to the ratio of the characteristics of the same color. In case of the shape coding with pictograms it is feasible to show all the pictograms of all the service once. In addition it is possible to size the pictograms or to adjust their opacity according to the ratio of characteristics for each pictogram. If the user filters services or zooms in so that the size of the displayed part is sufficient, the view changes to the small service boxes without names but with colors or small pictograms. If the

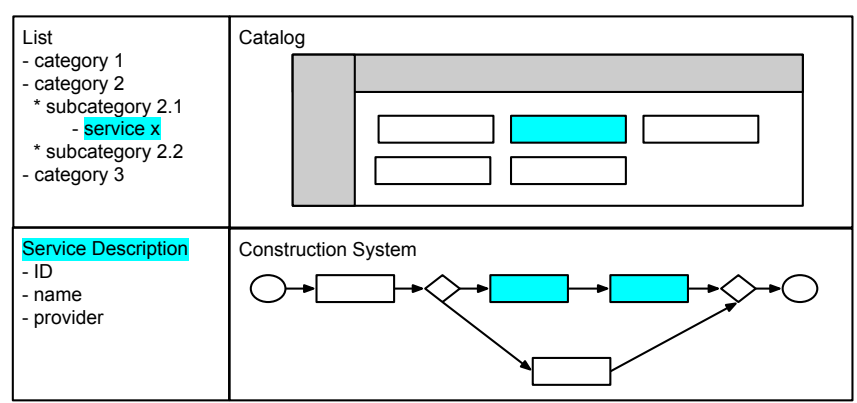

Fig. 8. Rough sketch of temporal fusion for service map concept.

user zooms further, the services will be shown normally. If the user wants to know the details of a service he can hover or click the service and the details will be shown in an overlay or a pop-up. This way it is possible to have a multitude of services shown in one category in a perceptible manner.

Another approach for enhancing the usability of the SM concept is temporal fusion, shown in Fig. 8. By a simultaneous and identical marking of the same aspect in different views, context-specific relations can be visualized.

\section{Conclusion and Future Prospects}

This paper presented an improvement of the information visualization for the service map (SM) concept by adding further dimensions. Starting with an introduction to information visualization a profound basis for the enhancement is given with an appropriate methodology, commonly accepted design guidelines, general challenges and a technical approach for implementing a visualization pipeline. Afterwards, the state of the art of the ESSENCE platform, as an representative example of a logistics cloud, was introduced as well as its current shortcomings, i.e. poor clarity in overview and difficulties in orchestration because of a poor user interface. Hence, the paper further introduced the SM concept as a combination of a catalog and a modular service construction system, in order to improve the current cloud platforms' disadvantages. Nevertheless, there were also some visual shortcomings for the SM concept. 
The particular contribution of the paper is a profound analysis of information visualization aspects and a derivation of an enhanced visualization approaches for the SM concept. Further 'dimensions' for the SM are realized by the possibility of adding categories represented by colors or pictograms. Finally, the paper determines suitable approaches by giving a guideline on choosing a particular visualization approach dependent on a given scale of measurement.

Future research prospects will address challenges of technically integrating the SM concept in logistics clouds like the ESSENCE platform. Furthermore, the realization of the introduced approach of temporal fusion will be implemented and analyzed towards its usability. Further research prospects comprise sophisticated visualization approaches. Potential ideas are situated in advanced field of free spatial service positioning in a graph theory oriented manner, whereas edge lengths could be related to similarity measures between services.

\section{REFERENCES}

[1] (2014) eurostat: Your key to european statistics. [Online]. Available: http://epp.eurostat.ec.europa.eu/portal/page/portal/eurostat/home/

[2] B. Adomavičius and Z. Lydeka, "Cooperation among the competitors in international cargo transportation sector: key factors to success," Engineering Economics, vol. 51, no. 1, pp. 80-90, 2007.

[3] Cruijssen, Franciscus Cornelis Andreas Maria, "Horizontal cooperation in transport and logistics," Ph.D. dissertation, Universiteit van Tilburg, Tilburg, 2006.

[4] R. Handfield, F. Straube, H.-C. Pfohl, and A. Wieland, Trends and Strategies in Logistics and Supply Chain Management: Embracing bal logistics complexity to drive market advantage, ser. Trends and strategies in logistics and supply chain management. Hamburg: DVV Media Group, 2013

[5] L. Terry. (2014) 2014 third-party logistics study: The state of logistics outsourcing: Results and findings of the 18th annual study. [Online]. Available: http://www.es.capgemini.com/resource-file-access/ resource/pdf/3pl\_study\_reportl_web\_version.pdf

[6] U. Arnold, J. Oberländer, and B. Schwarzbach, "Logical - development of cloud computing platforms and tools for logistics hubs and communities," in Computer Science and Information Systems (FedCSIS), 2012 Federated Conference on Computer Science and Information Systems, 2012, pp. 1083-1090.

[7] (2014) Essence: Easy eservices to shape and empower sme networks in central europe. [Online]. Available: http://www.essence-project.eu

[8] S. Liu, W. Cui, Y. Wu, and M. Liu, "A survey on information visualization: recent advances and challenges," The Visual Computer, 2014.

[9] M. Glöckner and A. Ludwig, "Towards a logistics service map: Support for logistics service engineering and management," in Pioneering solutions in supply chain performance management: Proceedings of the Hamburg International Conference of Logistics (HICL) 2013, ser. Reihe: Supply chain, logistics and operations management, T. Blecker, W. Kersten, and C. Ringle, Eds. Eul, 2013, vol. 17, pp. 309-324.
[10] M. Sedlmair, M. Meyer, and T. Munzner, "Design study methodology: Reflections from the trenches and the stacks," IEEE Transactions on Visualization and Computer Graphics, vol. 18, no. 12, pp. 2431-2440, 2012.

[11] A. Dix, "Introduction to information visualisation," in Information Retrieval Meets Information Visualization, ser. Lecture Notes in Computer Science, D. Hutchison, T. Kanade, J. Kittler, J. M. Kleinberg, F. Mattern, J. C. Mitchell, M. Naor, O. Nierstrasz, C. Pandu Rangan, B. Steffen, M. Sudan, D. Terzopoulos, D. Tygar, M. Y. Vardi, G. Weikum, M. Agosti, N. Ferro, P. Forner, H. Müller, and G. Santucci, Eds. Springer Berlin Heidelberg, 2013, vol. 7757, pp. 1-27.

[12] B. Shneiderman and C. Plaisant, Designing the user interface: Strategies for effective human-computer interaction, 4th ed. Boston: Pearson/Addison Wesley, 2004.

[13] P. Pirolli, Information foraging theory: Adaptive interaction with information, ser. Oxford series in human-technology interaction. Oxford and New York: Oxford University Press, 2007.

[14] S. Haroz and D. Whitney, "How capacity limits of attention influence information visualization effectiveness," IEEE Transactions on Visualization and Computer Graphics, vol. 18, no. 12, pp. 2402-2410, 2012.

[15] D. A. Keim, F. Mansmann, J. Schneidewind, and H. Ziegler, "Challenges in visual data analysis," in Tenth International Conference on Information Visualisation (IV'06), 2006, pp. 9-16.

[16] S. Eicker, T. Spies, and C. Kahl, "Software visualization in the context of service-oriented architectures," in 2007 4th IEEE International Workshop on Visualizing Software for Understanding and Analysis, 2007, pp. 108-111.

[17] H. Schumann and W. Müller, Visualisierung. Berlin and Heidelberg: Springer Berlin Heidelberg, 2000.

[18] F. Kohlmann and R. Alt, "Aligning service maps - a methodological approach from the financial industry," in Proceedings of the 42nd Annual Hawaii International Conference on System Sciences, R. H. Sprague, Ed. IEEE Computer Society Press, 2009, pp. 1-10.

[19] J. Kim, S. Lee, and Y. Park, "User-centric service map for identifying new service opportunities from potential needs: A case of app store applications," Creativity and Innovation Management, vol. 22, no. 3, pp. 241-264, 2013.

[20] S. Vaddi, H. Mohanty, and R. Shyamasundar, "Service maps in xml," in Proceedings of the CUBE International Information Technology Conference, V. Potdar, Ed. ACM, 2012, pp. 635-640.

[21] D. Kutscher and J. Ott, "Service maps for heterogeneous network environments," in MDM 2006 Japan. IEEE Computer Society, 2006.

[22] Mi Sun Ryu, Hong-Shik Park, and Sang-Chul Shin, "Qos class mapping over heterogeneous networks using application service map," in Networking, International Conference on Systems and International Conference on Mobile Communications and Learning Technologies, 2006. ICN, 2006, p. 13.

[23] T. Kohlborn, E. Fielt, A. Korthaus, and M. Rosemann, "Towards a service portfolio management framework," in ACIS2009 - Australian Conference on Information Systems, 2009, pp. 861-870.

[24] J. Fleischer, M. Herm, U. Homann, K. Peter, and K.-H. Sternemann, "Business capabilities als basis fähigkeitsorientierter konfigurationen," ZWF - Zeitschrift für wirtschaftlichen Fabrikbetrieb, vol. 100, no. 10 pp. 553-557, 2005.

[25] S. S. Stevens, "On the theory of scales of measurement," 1946.

[26] E. R. Tufte, Envisioning information, 5th ed. Cheshire and Conn: Graphics Press, 1995, c1990.

[27] M. Tkalcic, J. F. Tasic et al., "Colour spaces: perceptual, historical and applicational background," in Eurocon, 2003. 